\title{
Electromagnetic properties of carbonyl iron and their microwave absorbing characterization as filler in silicone rubber
}

\author{
YUPING DUAN*, GUOFANG LI, LIDONG LIU and SHUNHUA LIU \\ School of Material and Engineering, Dalian University of Technology, Dalian 116085, China
}

MS received 15 April 2009; revised 6 May 2009

\begin{abstract}
The complex permittivity, permeability and microwave-absorbing properties of rubber composites filled with carbonyl iron are measured at frequencies from 2-18 GHz. The results indicate that the reflection loss peak shifts towards low frequency region with increasing layer thickness or increasing weight concentration. The minimum reflection loss value of $-23.06 \mathrm{~dB}$ was obtained at $3.3 \mathrm{GHz}$ for the composites with $80 \%$ wt. These results show that the composites possess good microwave absorbing ability in both low- and highfrequency bands.
\end{abstract}

Keywords. Electromagnetic; carbonyl iron; absorbing characterization.

\section{Introduction}

With the development of radar, microwave communication technology and especially the need for anti-electromagnetic interference coatings, self-concealing technology and anechoic chamber, the study of electromagnetic wave absorbing materials has increased in recent years (Ghasemi et al 2006). People have been attracted by rubber radar absorbing material (RAM) for many years because of its special characteristic, which can make the RAM soft, flexible, and easy to be clipped (Feng et al 2006). Many studies have been carried out to investigate the effects of particulates and their volume percentages in composites on the absorption of electromagnetic waves to make absorbers (Kim et al 2004, 2009; Madina et al 2007). However, they have some drawbacks, such as great thickness, narrow absorptive bandwidth, etc.

The aim of this paper is to develop a novel thin microwave absorber with good absorbing performance in wide bandwidth and lightweight. So we investigated the microwave absorbing characterization of silicone rubber using carbonyl iron as filler. Carbonyl iron can be widely used in the field of electromagnetic shielding and absorbing materials (Kimura et al 2007; Yu et al 2008) in a wide frequency range because it has high Curie temperature, good temperature stabilization, the higher specific saturation magnetization intensity, high value of microwave permeability and dielectric constant. As a synthetic rubber, silicone rubber has excellent weathering resistance, resistance to aging, chemical resistance and insulating properties, and it has good compatibility with many kinds of fillers. Thereby, it is favourable to be the matrix of the

\footnotetext{
*Author for correspondence (duanyp@dlut.edu.cn)
}

rubber radar absorbing material. Furthermore, the particle size of the carbonyl iron is $<5 \mu \mathrm{m}$, and the micron filler can strengthen the silicone rubber.

In this study, the electromagnetic parameters and the microwave absorbing property of carbonyl iron was studied using silicone rubber as the base material. The results of the study show that carbonyl iron is a type of magnetic loss material and performs well in both lowand high-frequency bands.

\section{Experimental}

\subsection{Materials}

Carbonyl iron powder (diameter, $3.4 \mu \mathrm{m}$ ) was procured from Jiangyou Hebao Nanophase Materials Corporation. The elastomer used was silicone rubber (density, $0.93 \mathrm{~g} / \mathrm{cm}^{3}$ ), which was supplied by Zhonglan Chenguang Chemistry Research Institute. All other reagents were of analytical grade and used without purification.

\subsection{Fabrication of test pieces}

The fillers were individually blended in a proportion with vulcanization agents in silicone rubber on a two-roll mill at ambient temperature with a rotor speed of 22 and $25 \mathrm{rpm}$ (back and forward), according to classical procedures used in rubber industry. The blends were compression moulded at $170^{\circ} \mathrm{C}$. Moulding was carried out in a hydraulic press at a pressure of $15.5 \mathrm{MPa}$, obtaining vulcanized test specimens of $20 \times 20 \mathrm{~cm}$ for reflectivity measurements. The curing times were determined at $170^{\circ} \mathrm{C}$ for $12 \mathrm{~min}$. 


\subsection{Measurement of properties}

Study of morphologies of carbonyl iron powder was conducted by scanning electron microscopy (SEM: JSM$5600 \mathrm{LV}$ ). The absorbing effectiveness of wave absorbing material was denoted by the reflectivity, $R$, which was expressed as

$$
R=20 \log \left|E_{\mathrm{r}} / E_{\mathrm{i}}\right|(\mathrm{dB}),
$$

where $E_{\mathrm{r}}$ and $E_{\mathrm{i}}$ refer to the electric field strength of the incident and reflective electromagnetic wave, respectively. The arched test method was used in this study and is shown in figure 1. The set up was connected to a HP8720B microwave network analyzer. A calibration kit was first used to calibrate the test system and the specimens were tested in the frequency range $2-18 \mathrm{GHz}$. The electromagnetic parameters of carbonyl iron powder were tested using the coaxial cable method.

\section{Results and discussion}

\subsection{Morphology of carbonyl iron particles}

The SEM topography of carbonyl iron particle shows that carbonyl iron is of spherical morphology (figure 2). It could be found that the particle size of the carbonyl iron powder ranged from $1-4 \mu \mathrm{m}$, most of the particles are in the range of $3 \sim 4 \mu \mathrm{m}$.

\subsection{Electromagnetic property of carbonyl iron powder}

Figures 3 and 4 show the electromagnetic parameters of carbonyl iron powder. From figure 4, we can see that the permeability of carbonyl iron have maximum values at $2 \mathrm{GHz}$. The dispersion effect of the real part, $\mu^{\prime}$ and the imaginary part, $\mu^{\prime \prime}$, of the complex permeability of carbonyl iron is inconspicuous for its little variation in the whole frequency range studied, the effect can lead to reduction of the matching thickness (Cao et al 2003).

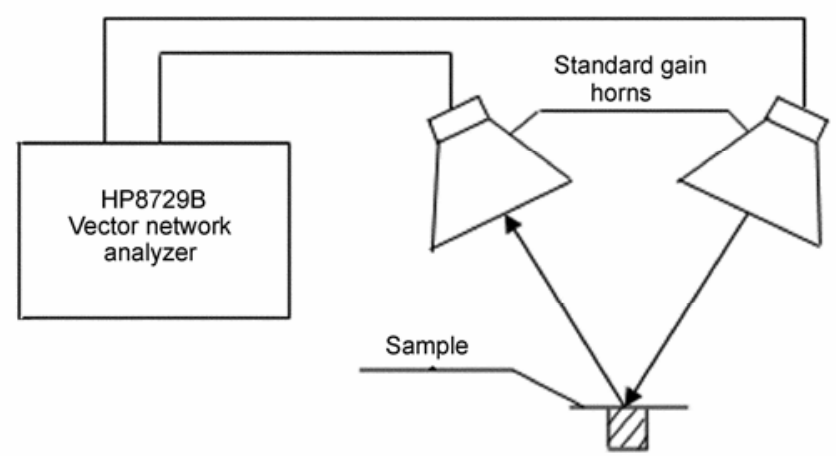

Figure 1. Set-up for arched test method.
The $\varepsilon^{\prime}$ and $\varepsilon^{\prime \prime}$ values of carbonyl iron decrease with increase in frequency in low frequency range $(2 \sim 8 \mathrm{GHz})$. The consistency of the dielectric permittivity as the frequency is increased in high frequency region $(8 \sim 18 \mathrm{GHz})$ is simply due to the fact that the polarization of the dielectric dipoles in the RAM are in-phase with the oscillation of the electric field vector of the transverse electromagnetic (TEM) wave (Feng et al 2007). It can be deduced that carbonyl iron is a kind of magnetic loss material. There is a strong interaction between carbonyl iron particle with the ferromagnetic property and microwaves because of mechanisms such as electron energylevel transitions in metals, relative vibration of atoms in optical waves, and changes in the atomic rotation energy level and atomic magnetic energy level. Therefore, carbonyl iron particles have a strong ability to absorb electromagnetic wave.

For a single-layered electromagnetic absorber with lower electric conductivity backed with a metal plate, its input impedance, $Z_{\text {in }}$, normalized by the impedance of free space, $Z_{0}$, is expressed as

$$
Z=\frac{Z_{\text {in }}}{Z_{0}}=\sqrt{\frac{\mu_{\mathrm{r}}}{\varepsilon_{\mathrm{r}}}} \tan h\left(j \frac{2 \pi d}{\lambda_{0}} \cdot \sqrt{\mu_{\mathrm{r}} \varepsilon_{\mathrm{r}}}\right),
$$

where $\mu_{\mathrm{r}}, \varepsilon_{\mathrm{r}}, d$ and $\lambda_{0}$ represent the relative complex permeability, permittivity, thickness and wavelength of the incident wave in free space, respectively. So the reflection coefficient with respect to a normally incident plane wave is

$$
R=20 \lg \left|\frac{Z-1}{Z+1}\right|
$$

From (1) and figures 3 and 4 we can see that the electromagnetic property of carbonyl iron powder can enhance the absorbency of the composite. Carbonyl iron have huge specific surface area, and there is a forceful

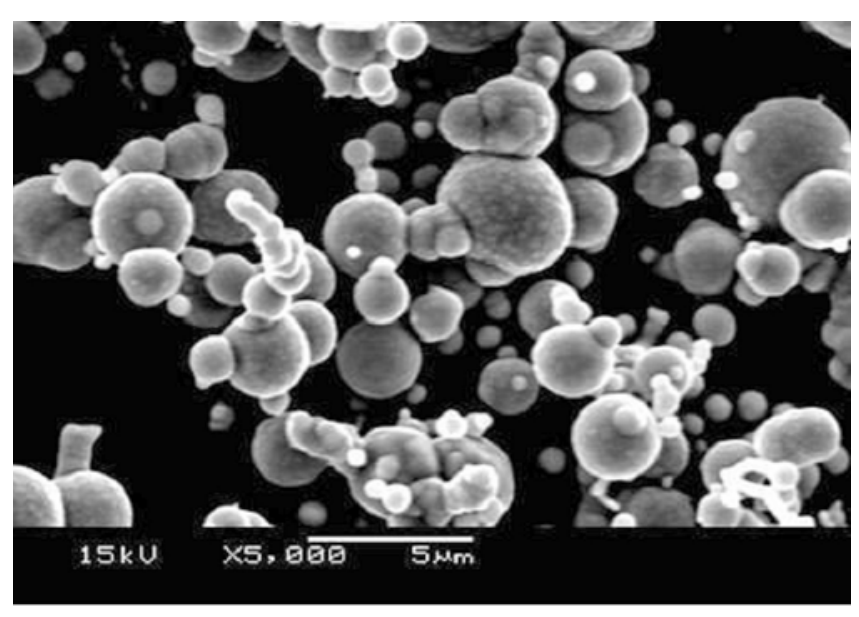

Figure 2. SEM of carbonyl iron powder. 
interaction between carbonyl iron particles and incident wave, so it can effectively attenuate the electromagnetic wave. As a result, carbonyl iron will hopefully be an absorbent with wide bandwidth and strong absorption.

\subsection{Absorbing property of carbonyl iron composite}

3.3a Influence of carbonyl iron powder filling ratio: Four types of samples were prepared with the carbonyl iron weight fraction of $50 \% \mathrm{wt}$., $60 \% \mathrm{wt}$., $70 \% \mathrm{wt}$. and $80 \%$ wt. The samples were of $4 \mathrm{~mm}$ thickness and were labeled as $1 \# \sim 4 \#$.

Figure 5 shows the frequency dependence of the reflectivity in $2 \sim 18 \mathrm{GHz}$. Results show that with increase in carbonyl iron filling ratio, the reflectivity increases gradually and the absorption peak value shifts to lower frequency band, which can also be seen in table 1 .

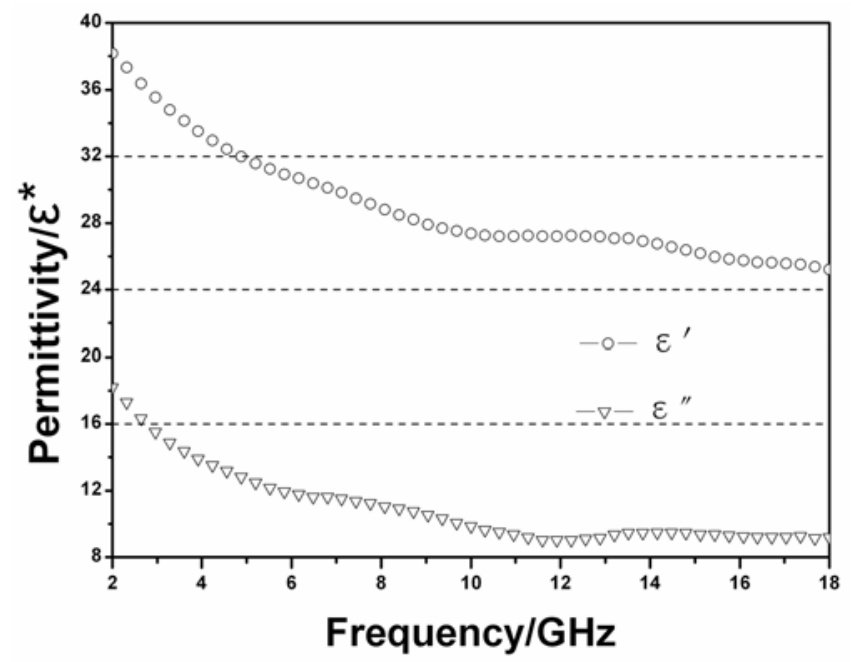

Figure 3. The permittivity curve of carbonyl iron.

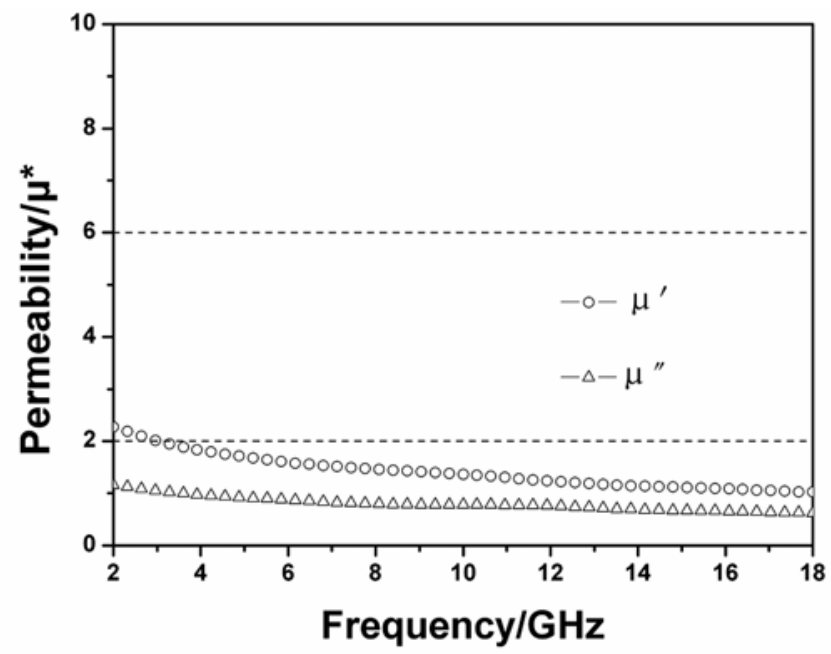

Figure 4. The permeability curve of carbonyl iron.
The reflectivity of $1 \#$ sample has only one peak value of $-7.36 \mathrm{~dB}$ at $5.5 \mathrm{GHz}$, but it can be deduced from the curve that there must have been another absorption peak at the frequency band above $18 \mathrm{GHz}$. 2 \# is better than 1 , it has one peak value of $-14.48 \mathrm{~dB}$ at $4.9 \mathrm{GHz}$ and a second peak value of $-11.42 \mathrm{~dB}$ at $15.9 \mathrm{GHz}$. 3\# has shifted its absorption peak to a lower frequency of $3.4 \mathrm{GHz}$ and with a value of $-18.92 \mathrm{~dB}$, and the second absorption peak also shifts to a lower frequency of $-10 \cdot 28 \mathrm{~dB}$ at 11.2 GHz. 4\# has the approximate trend with 3\#. The reflectivity peak of $4 \#$ shifts to a lower frequency than any other samples and it gets a maximum reflectivity of $-23.06 \mathrm{~dB}$ at $3.3 \mathrm{GHz}$, and the frequency bandwidth at which the reflection loss is less than $-10 \mathrm{~dB}$ is $0.8 \mathrm{GHz}$. It has the second peak value of $-6.67 \mathrm{~dB}$ at $10.8 \mathrm{GHz}$. With the carbonyl iron content increasing, the imaginary part and the loss tangent of the composite also increased (Qing et al 2009), according to (1), the absorbency of the composite can obviously be raised.

3.3b Influence of sample thickness: Figure 6 indicates the absorbing characteristics of $2 \mathrm{~mm}$ thick samples in $2 \sim 18 \mathrm{GHz}$ with carbonyl iron loading of $50 \%$ wt., $60 \%$ wt., $70 \%$ wt. and $80 \%$ wt., the samples being noted as $5 \# \sim 8 \#$, respectively.

Figure 6 indicates that there is one absorption peak for each sample in the frequency band of $2 \sim 18 \mathrm{GHz}$ and to the samples of the same CIP filling proportion, the decreasing of thickness shifts the absorbing peak value to higher frequency band, which is also shown in table 1 .

Take 4\# and 8\# with the same carbonyl iron loading of $80 \%$ wt for example, when the thickness of the sample changes from $4-2 \mathrm{~mm}$, the absorbing peak values have been changed from $3 \cdot 3-5.9 \mathrm{GHz}$. From the trend of absorbing curve of $8 \#$, it is obvious that the reflectivity decreases drastically with the increasing of frequency

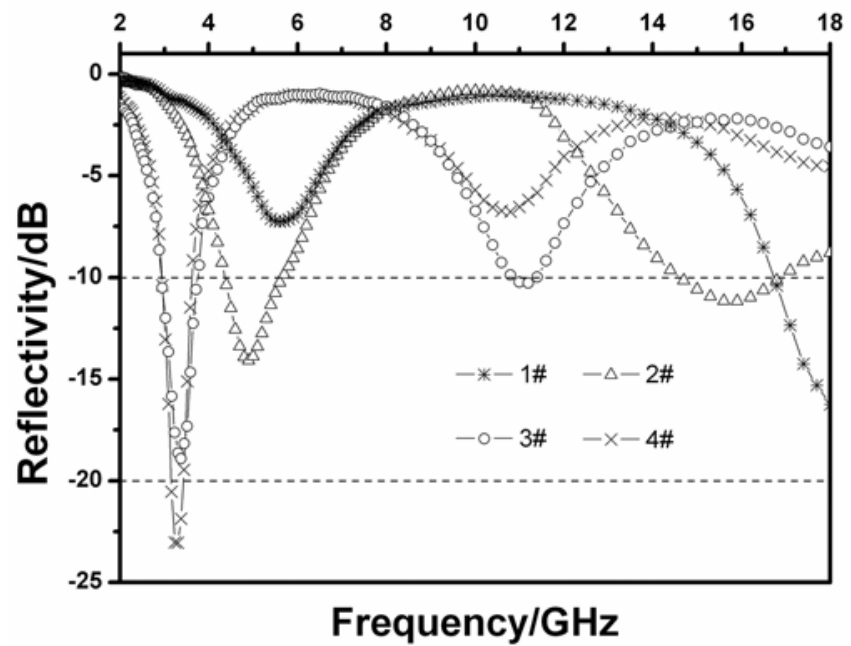

Figure 5. Reflectivity of carbonyl iron with thickness of $4 \mathrm{~mm}$. 
Table 1. Absorbing peak frequency and values for carbonyl iron composites

\begin{tabular}{lrrrr}
\hline & \multicolumn{3}{c}{ Carbonyl iron filling proportion } \\
\cline { 2 - 5 } Thickness $(\mathrm{mm})$ & $50 \% \mathrm{wt}$. & $60 \% \mathrm{wt}$. & $70 \% \mathrm{wt}$. & $80 \% \mathrm{wt}$. \\
\hline 2 & $9.8 \mathrm{GHz}$ & $8.4 \mathrm{GHz}$ & $6.0 \mathrm{GHz}$ & $5.9 \mathrm{GHz}$ \\
& $(-9.26 \mathrm{~dB})$ & $(-16.26 \mathrm{~dB})$ & $(-20.5 \mathrm{~dB})$ & $(-20.48 \mathrm{~dB})$ \\
4 & $5.5 \mathrm{GHz}$ & $4.9 \mathrm{GHz}$ & $3.4 \mathrm{GHz}$ & $3.3 \mathrm{GHz}$ \\
& $(-7.36 \mathrm{~dB})$ & $(-14.48 \mathrm{~dB})$ & $(-18.92 \mathrm{~dB})$ & $(-23.06 \mathrm{~dB})$ \\
& - & $15.9 \mathrm{GHz}$ & $11.2 \mathrm{GHz}$ & $10.8 \mathrm{GHz}$ \\
& & $(-11.42 \mathrm{~dB})$ & $(-10.28 \mathrm{~dB})$ & $(-6.67 \mathrm{~dB})$ \\
\hline
\end{tabular}

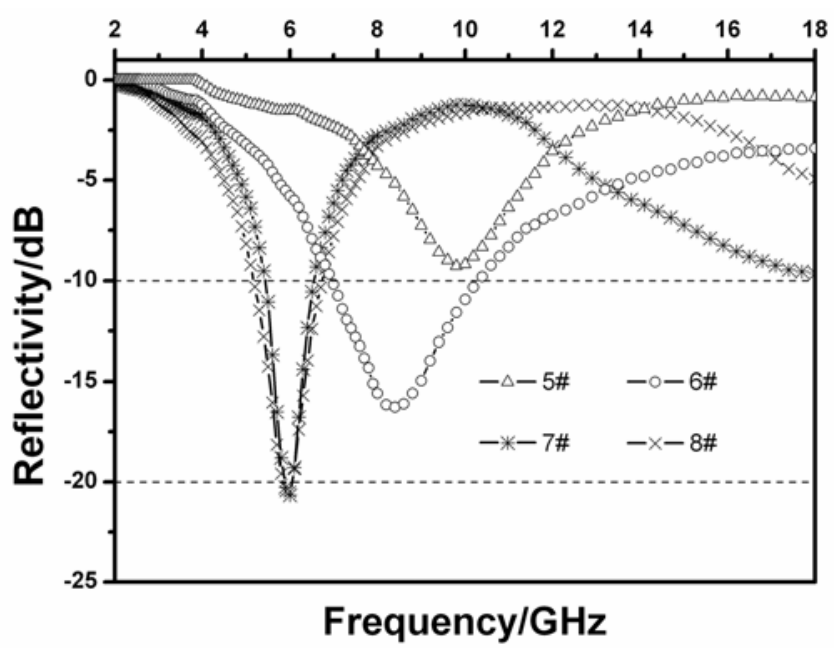

Figure 6. Reflectivity of carbonyl iron with thickness of $2 \mathrm{~mm}$.

over $12 \mathrm{GHz}$, and we can see that there must have been another peak value at some frequency higher than $18 \mathrm{GHz}$. It is known that thickness has an obvious influence on electromagnetic wave absorbing performance, but their relationship is not simply linear (Oh et al 2004). In a certain range, the number of electromagnetic wave scattering and reflection is increased in the composites with increase in thickness, which also increases the spread optical length, so the thickness increase have some effect on the attenuation of the electromagnetic wave.

Compare $3 \#$ and $4 \#$ in figure 5, 7\# and 8 \# in figure 6 , we can see that they almost have the same absorbing curve. According to the classical statistical percolation model, when the weight concentration achieves $70 \%$, the volume ratio of the particles has reached the percolation threshold, the conductive network was completed, the transmission of the composite was diminished, but the reflectivity was augmented. So the surface reflection of the electromagnetic wave was larger, electromagnetic wave cannot enter the composite to have effective attenuation. So the increase of the particle content did not have much effect on the absorbing performance.

\section{Conclusions}

Carbonyl iron powder is a kind of magnetic loss absorbent of electromagnetic microwave. With the increase of carbonyl iron powder filling and the composite thickness, the absorption peak of carbonyl iron powder/silicone rubber composite shifts to lower frequency region. When the weight concentration achieves $70 \%$, the increase of the particles contents did not have much effect on the absorbing performance.

\section{Acknowledgements}

The authors acknowledge the support from the National Natural Science Foundation of China (No. 51007005), The Research Fund for the Doctoral Program of Higher Education of China (20090041120038), The Aero Science Foundation of China (20095463008), and The Fundamental Research Funds for the Central Universities and the Growth Foundation for the Youth Teachers of the Dalian University of Technology.

\section{References}

Cao M Sh, Qin R R and Qiu Ch J 2003 Mater. Des. 24391

Feng Y B, Qiu T and Shen C Y 2006 IEEE Trans. Magn. 42 363

Feng Y B, Qiu T and Shen C Y 2007 J. Magn. Magn. Mater. 3188

Ghasemi A, Hossienpour A and Morisako A 2006 J. Magn. Magn. Mater. 302429

Kim M S, Min E H and Koh J G 2009 J. Magn. Magn. Mater. 321581

Kim S S, Kim S T and Ahn J M 2004 J. Magn. Magn. Mater. 27139

Kimura S, Kato T and Hyodo T 2007 J. Magn. Magn. Mater. 312181

Madina A A, Alexander V L and Natalia E K 2007 Composites Part A 382471

Oh J H, Oh K S and Kim C G 2004 Composites Part B 3549

Qing Y Ch, Zhou W Ch and Luo F 2009 J. Magn. Magn. Mater. 32125

Yu M X, Li X Ch and Gong R Zh 2008 J. Alloys Compd 456452 